\title{
AGGREGATION AND CAPITAL ALLOCATION FORMULAS FOR BIVARIATE DISTRIBUTIONS
}

DOI:

$10.1017 /$ S0269964817000377

\section{Document Version}

Accepted author manuscript

Link to publication record in Manchester Research Explorer

\section{Citation for published version (APA):}

Nadarajah, S., Chu, J., \& Jiang, X. (2017). AGGREGATION AND CAPITAL ALLOCATION FORMULAS FOR BIVARIATE DISTRIBUTIONS. Probability in the Engineering and Informational Sciences, 1-11.

https://doi.org/10.1017/S0269964817000377

\section{Published in:}

Probability in the Engineering and Informational Sciences

\section{Citing this paper}

Please note that where the full-text provided on Manchester Research Explorer is the Author Accepted Manuscript or Proof version this may differ from the final Published version. If citing, it is advised that you check and use the publisher's definitive version.

\section{General rights}

Copyright and moral rights for the publications made accessible in the Research Explorer are retained by the authors and/or other copyright owners and it is a condition of accessing publications that users recognise and abide by the legal requirements associated with these rights.

\section{Takedown policy}

If you believe that this document breaches copyright please refer to the University of Manchester's Takedown Procedures [http://man.ac.uk/04Y6Bo] or contact uml.scholarlycommunications@manchester.ac.uk providing relevant details, so we can investigate your claim.

\section{OPEN ACCESS}




\title{
Aggregation and capital allocation formulas for bivariate distributions
}

\author{
by
}

\author{
Saralees Nadarajah, Jeffrey Chu and Xiao Jiang \\ University of Manchester, Manchester M13 9PL, UK, \\ email: mbbsssn2@manchester.ac.uk
}

\begin{abstract}
Cossette, Marceau and Perreault [Insurance: Mathematics and Economics, 64, 2015, 214-224] derived formulas for aggregation and capital allocation based on risks following two bivariate exponential distributions. Here, we derive formulas for aggregation and capital allocation for thirty six mostly commonly known families of bivariate distributions. This collection of formulas could be a useful reference for financial risk management.
\end{abstract}

Keywords: Closed form; Risk; Sum

\section{Introduction}

Results related to the sum of dependent risks are of interest in the calculation of the overall capital charge for a portfolio of risks, the evaluation and analysis of risk measures for decision making, and strategic planning require the knowledge of the cumulative distribution function of the sum of dependent random variables (Cossette et al., 2015). Risk measures like Value at Risk and Tail-Value at Risk can be used for these purposes.

In recent years, several closed form expressions for the distribution of aggregate risks, its TVaR and TVaR based allocations have been developed, based on an allocation method due to Tasche (1999). These expressions are based on a given joint distribution between the components of a portfolio. The joint distributions considered so far are the: multivariate normal distribution (Panjer, 2002); multivariate elliptical distributions (Landsman and Valdez, 2003; Dhaene et al., 2008); multivariate gamma distribution (Furman and Landsman, 2005); multivariate Tweedie distribution (Furman and Landsman, 2008); multivariate Pareto distribution (Chiragiev and Landsman, 2007); Farlie-Gumbel-Morgenstern copula (Barges et al., 2009); Farlie-Gumbel-Morgenstern copula with mixed Erlang marginals (Cossette et al., 2013); multivariate compound distributions (Cossette et al., 2012); bivariate exponential and bivariate mixed Erlang distributions (Cossette et al., 2015).

The aim of this note is to derive expressions for the distribution of aggregate risks, its TVaR and TVaR based allocations for a comprehensive collection of bivariate distributions. We consider thirty six families of bivariate distributions each defined on $(0, \infty) \times(0, \infty)$ or $(\beta, \infty) \times(\beta, \infty)$ for some $\beta>0$. They include mixtures of independent exponential distributions, Mirhosseini et al. (2015)'s bivariate exponential distribution, Crovelli (1973)'s bivariate exponential distribution, Gumbel's bivariate exponential distribution, Lawrance and Lewis' (1980) bivariate exponential distribution, Block and Basu (1976)'s bivariate exponential distribution, Arnold and Strauss (1991)'s bivariate exponential distribution, beta exponential distribution in equation (8.41) of Balakrishnan and Cai (2009), mixtures of independent gamma distributions with integer shape parameters, mixtures of independent gamma distributions with real shape parameters, Nadarajah and Gupta (2006)'s bi- 
variate gamma distribution with equal scale parameters, Nadarajah and Gupta (2006)'s bivariate gamma distribution with unequal scale parameters, Nagar and Sepulveda-Murillo (2011)'s bivariate confluent hypergeometric distribution, Becker and Roux (1981)'s bivariate gamma distribution, Mohsin et al. (2013)'s bivariate gamma distribution, Cheriyan (1941)'s bivariate gamma distribution, Dussauchoy and Berland (1975)'s bivariate gamma distribution, mixtures of independent two piece gamma distributions, beta Stacey distribution in equation (5.38) of Balakrishnan and Cai (2009), Mardia (1970)'s bivariate distributions, bivariate Liouville distributions, bivariate equilibrium distributions due to Unnikrishnan Nair and Sankaran (2014), Chacko and Thomas (2007)'s bivariate Pareto distribution, bivariate Pareto distribution in equation (10.68) of Balakrishnan and Cai (2009), bivariate Pareto distribution with equal scale parameters, bivariate Pareto distribution with unequal scale parameters, mixtures of independent Pareto distributions, mixtures of bivariate Pareto distributions with equal scale parameters, mixtures of bivariate Pareto distributions with unequal scale parameters, generalized bivariate Pareto distribution, Lee and Cha (2014)'s bivariate distribution and truncated bivariate normal distribution. We have not considered the bivariate normal or other distributions defined over $(-\infty, \infty) \times(-\infty, \infty)$, as they have already been considered by others.

\section{Mathematical notation}

Let $(X, Y)$ be non-negative continuous risks with joint probability density function $f(x, y)$. Let $S=X+Y$ denote the aggregated risk. We are interested in: the probability density function of $S$ given by

$$
f_{S}(s)=\int_{0}^{s} f(y, s-y) d y ;
$$

the cumulative distribution function of $S$ given by

$$
F_{S}(s)=\int_{0}^{s} f_{S}(y) d y
$$

the truncated expectation of $S$ given by

$$
E\left[S 1_{\{S>b\}}\right]=\int_{b}^{\infty} s f_{S}(s) d s ;
$$

the contribution of each risk to the aggregated risk given by

$$
E\left[X 1_{\{S>b\}}\right]=\int_{b}^{\infty} g_{X, S}(s) d s,
$$

where

$$
g_{X, S}(s)=\int_{0}^{s} x f(x, s-x) d x .
$$

We derive expressions for (1)-(5) for thirty six bivariate distributions.

The derived expressions given in Section 3 involve several special functions, including the gamma function defined by

$$
\Gamma(a)=\int_{0}^{\infty} t^{a-1} \exp (-t) d t
$$


the incomplete gamma function defined by

$$
\gamma(a, x)=\int_{0}^{x} t^{a-1} \exp (-t) d t
$$

the complementary incomplete gamma function defined by

$$
\Gamma(a, x)=\int_{x}^{\infty} t^{a-1} \exp (-t) d t
$$

the error function defined by

$$
\operatorname{erf}(x)=\frac{2}{\sqrt{\pi}} \int_{0}^{x} \exp \left(-t^{2}\right) d t
$$

the beta function defined by

$$
B(a, b)=\int_{0}^{1} t^{a-1}(1-t)^{b-1} d t
$$

the incomplete beta function defined by

$$
B_{x}(a, b)=\int_{0}^{x} t^{a-1}(1-t)^{b-1} d t
$$

the standard normal distribution function defined by

$$
\Phi(x)=\frac{1}{\sqrt{2 \pi}} \int_{-\infty}^{x} \exp \left(-\frac{t^{2}}{2}\right) d t
$$

the confluent hypergeometric function defined by

$$
{ }_{1} F_{1}(a ; b ; x)=\sum_{k=0}^{\infty} \frac{(a)_{k}}{(b)_{k}} \frac{x^{k}}{k !} ;
$$

the Tricomi confluent hypergeometric function defined by

$$
U(a, b, x)=\frac{\Gamma(b-1)}{\Gamma(a)} x^{1-b}{ }_{1} F_{1}(a-b+1 ; 2-b ; x)+\frac{\Gamma(1-b)}{\Gamma(a-b+1)}{ }_{1} F_{1}(a ; b ; x) ;
$$

the Gauss hypergeometric function defined by

$$
{ }_{2} F_{1}(a ; b ; x)=\sum_{k=0}^{\infty} \frac{(a)_{k}(b)_{k}}{(c)_{k}} \frac{x^{k}}{k !} ;
$$

the Appell hypergeometric function of the first kind of two variables defined by

$$
F_{1}(a, b, c, d ; x, y)=\sum_{m=0}^{\infty} \sum_{n=0}^{\infty} \frac{(a)_{m+n}(b)_{m}(c)_{n} x^{m} y^{n}}{(d)_{m+n} m ! n !}
$$

and the degenerate hypergeometric series of two variables defined by

$$
\Phi_{1}(a, b, c, x, y)=\sum_{m=0}^{\infty} \sum_{n=0}^{\infty} \frac{(a)_{m+n}(b)_{n} x^{m} y^{n}}{(c)_{m+n} m ! n !},
$$

where $(e)_{k}=e(e+1) \cdots(e+k-1)$ denotes the ascending factorial.

In-built routines for computing these special functions are available in packages like Matlab, Maple and Mathematica. For example, Erf[x], Gamma[a, x], Beta[a, b], Beta[x, a, b], Hypergeometric1F1[a, b, x], Hypergeometric2F1[a, b, c, x] and AppellF1[a, b, c, d, x, y] in Mathematica compute the error, complementary incomplete gamma, beta, incomplete beta, confluent hypergeometric, Gauss hypergeometric and Appell hypergeometric functions. Mathematica allows for arbitrary precision, so the accuracy of computations is not an issue. 


\section{The collection}

Here, we give expressions for (1)-(5) for thirty six bivariate distributions. Their derivations are routine and are not presented. The detailed derivations can be obtained from the corresponding author.

\section{Mixtures of independent exponential distributions:}

$$
\begin{aligned}
& f(x, y)=\sum_{k=1}^{m} C_{k} \exp \left(-\alpha_{k} x-\beta_{k} y\right), \\
& f_{S}(s)=\sum_{k=1}^{m} \frac{C_{k}}{\beta_{k}-\alpha_{k}}\left[\exp \left(-\alpha_{k} s\right)-\exp \left(-\beta_{k} s\right)\right], \\
& F_{S}(s)=\sum_{k=1}^{m} \frac{C_{k}}{\beta_{k}-\alpha_{k}}\left[\frac{1-\exp \left(-\alpha_{k} s\right)}{\alpha_{k}}-\frac{1-\exp \left(-\beta_{k} s\right)}{\beta_{k}}\right], \\
& E\left[S 1_{\{S>b\}}\right]=\sum_{k=1}^{m} \frac{C_{k}}{\beta_{k}-\alpha_{k}}\left[\frac{\left(b \alpha_{k}+1\right) \exp \left(-b \alpha_{k}\right)}{\alpha_{k}^{2}}-\frac{\left(b \beta_{k}+1\right) \exp \left(-b \beta_{k}\right)}{\beta_{k}^{2}}\right], \\
& g_{X, S}(s)=\sum_{k=1}^{m} C_{k} \exp \left(-\beta_{k} s\right)\left[\frac{1-\exp \left(\beta_{k} s-\alpha_{k} s\right)}{\left(\alpha_{k}-\beta_{k}\right)^{2}}-\frac{s \exp \left(\beta_{k} s-\alpha_{k} s\right)}{\alpha_{k}-\beta_{k}}\right], \\
& E\left[X 1_{\{S>b\}}\right]=\sum_{k=1}^{m} C_{k}\left[-\frac{\left(\alpha_{k} b+1\right) \exp \left(-\alpha_{k} b\right)}{\alpha_{k}^{2}\left(\alpha_{k}-\beta_{k}\right)}+\frac{\exp \left(-\beta_{k} b\right)}{\beta_{k}\left(\alpha_{k}-\beta_{k}\right)^{2}}-\frac{\exp \left(-\alpha_{k} b\right)}{\alpha_{k}\left(\alpha_{k}-\beta_{k}\right)^{2}}\right]
\end{aligned}
$$

for $\alpha_{k}>0, \beta_{k}>0, x>0$ and $y>0$.

Mirhosseini et al. (2015)'s bivariate exponential distribution:

$$
\begin{aligned}
& f(x, y)=\lambda^{2} \alpha \exp (-\lambda x-\lambda y)[1-\alpha \exp (-\lambda x-\lambda y)][1-\exp (-\lambda x-\lambda y)]^{\alpha-2}, \\
& f_{S}(s)=\lambda^{2} \alpha s \exp (-\lambda s)[1-\alpha \exp (-\lambda s)][1-\exp (-\lambda s)]^{\alpha-2}, \\
& F_{S}(s)=\alpha \sum_{k=0}^{\infty}\left(\begin{array}{c}
\alpha-2 \\
k
\end{array}\right) \frac{(-1)^{k}}{(k+1)^{2}}[1-(1+(k+1) \lambda s) \exp (-(k+1) \lambda s)] \\
& \quad-\alpha^{2} \sum_{k=0}^{\infty}\left(\begin{array}{c}
\alpha-2 \\
k
\end{array}\right) \frac{(-1)^{k}}{(k+2)^{2}}[1-(1+(k+2) \lambda s) \exp (-(k+2) \lambda s)], \\
& E\left[S 1_{\{S>b\}}\right]=\frac{2 \alpha}{\lambda} \sum_{k=0}^{\infty}\left(\begin{array}{c}
\alpha-2 \\
k
\end{array}\right) \frac{(-1)^{k}}{(k+1)^{3}}\left(1+(k+1) \lambda b+\frac{(k+1)^{2} \lambda^{2} b^{2}}{2}\right) \exp (-(k+1) \lambda b) \\
& \quad-\frac{2 \alpha^{2}}{\lambda} \sum_{k=0}^{\infty}\left(\begin{array}{c}
\alpha-2 \\
k
\end{array}\right) \frac{(-1)^{k}}{(k+2)^{3}}\left(1+(k+2) \lambda b+\frac{(k+2)^{2} \lambda^{2} b^{2}}{2}\right) \exp (-(k+2) \lambda b), \\
& g_{X, S}(s)=2^{-1} \lambda^{2} \alpha s^{2} \exp (-\lambda s)[1-\alpha \exp (-\lambda s)][1-\exp (-\lambda s)]^{\alpha-2}, \\
& E\left[X 1_{\{S>b\}}\right]=\frac{\alpha}{\lambda} \sum_{k=0}^{\infty}\left(\begin{array}{c}
\alpha-2 \\
k
\end{array}\right) \frac{(-1)^{k}}{(k+1)^{3}}\left(1+(k+1) \lambda b+\frac{(k+1)^{2} \lambda^{2} b^{2}}{2}\right) \exp (-(k+1) \lambda b) \\
& \quad-\frac{\alpha^{2}}{\lambda} \sum_{k=0}^{\infty}\left(\begin{array}{c}
\alpha-2 \\
k
\end{array}\right) \frac{(-1)^{k}}{(k+2)^{3}}\left(1+(k+2) \lambda b+\frac{(k+2)^{2} \lambda^{2} b^{2}}{2}\right) \exp (-(k+2) \lambda b)
\end{aligned}
$$

for $\alpha>0, \lambda>0, x>0$ and $y>0$. 


\section{Crovelli (1973)'s bivariate exponential distribution:}

$$
\begin{aligned}
& f(x, y)= \begin{cases}\alpha \beta \exp (-\beta y)[1-\exp (-\alpha x)], & \text { if } 0 \leq \alpha x \leq \beta y \\
\alpha \beta \exp (-\beta x)[1-\exp (-\beta y)], & \text { if } 0 \leq \beta y \leq \alpha x\end{cases} \\
& f_{S}(s)=(\alpha+\beta) \exp \left(-\frac{\alpha \beta s}{\alpha+\beta}\right)+\frac{\alpha^{2}}{\alpha-\beta} \exp (-\beta s)-\frac{\beta^{2}}{\alpha-\beta} \exp (-\alpha s), \\
& F_{S}(s)=\frac{(\alpha+\beta)^{2}}{\alpha \beta}\left[1-\exp \left(-\frac{\alpha \beta s}{\alpha+\beta}\right)\right]+\frac{\alpha^{2}}{\beta(\beta-\alpha)}[1-\exp (-\beta s)] \\
& -\frac{\beta^{2}}{\alpha(\beta-\alpha)}[1-\exp (-\alpha s)] \text {, } \\
& E\left[S 1_{\{S>b\}}\right]=\frac{(\alpha+\beta)^{3}}{(\alpha \beta)^{2}} \exp \left(-\frac{\alpha \beta b}{\alpha+\beta}\right)\left[1+\frac{\alpha \beta b}{\alpha+\beta}\right]+\frac{\alpha^{2}}{\beta(\alpha-\beta)} \exp (-\beta b) \\
& -\frac{\beta^{2}}{\alpha(\alpha-\beta)} \exp (-\alpha b) \\
& g_{X, S}(s)=\frac{\beta^{2}}{\alpha-\beta} s \exp (-\alpha s)+\frac{\beta^{2}(2 \alpha-\beta)}{\alpha(\alpha-\beta)^{2}} \exp (-\alpha s)+\frac{\alpha^{2}(\alpha-2 \beta)}{\beta(\alpha-\beta)^{2}} \exp (-\beta s) \\
& +\beta s \exp \left(-\frac{\alpha \beta s}{\alpha+\beta}\right)+\frac{-\alpha^{4}+2 \alpha^{3}+\beta^{4}-2 \alpha \beta^{3}}{\alpha \beta(\alpha-\beta)^{2}} \exp \left(-\frac{\alpha \beta s}{\alpha+\beta}\right), \\
& E\left[X 1_{\{S>b\}}\right]=\frac{\beta^{2}}{\alpha^{2}(\alpha-\beta)}(1+\alpha b) \exp (-\alpha b)+\frac{\beta^{2}(2 \alpha-\beta)}{\alpha^{2}(\alpha-\beta)^{2}} \exp (-\alpha b) \\
& +\frac{\alpha^{2}(\alpha-2 \beta)}{\beta^{2}(\alpha-\beta)^{2}} \exp (-\beta b)+\frac{(\alpha+\beta)^{2}}{\alpha^{2} \beta}\left(1+\frac{\alpha \beta b}{\alpha+\beta}\right) \exp \left(-\frac{\alpha \beta b}{\alpha+\beta}\right) \\
& +\frac{(\alpha+\beta)\left(-\alpha^{4}+2 \alpha^{3}+\beta^{4}-2 \alpha \beta^{3}\right)}{(\alpha \beta)^{2}(\alpha-\beta)^{2}} \exp \left(-\frac{\alpha \beta b}{\alpha+\beta}\right)
\end{aligned}
$$

for $\alpha>0, \beta>0, x>0$ and $y>0$.

\section{Gumbel's bivariate exponential distribution:}

$$
\begin{aligned}
& f(x, y)= {[(1+\theta x)(1+\theta y)-\theta] \exp (-x-y-\theta x y), } \\
& f_{S}(s)=-\mathrm{i} \theta^{3 / 2} \sqrt{\pi} s^{2} \exp \left(-s-\frac{\theta s^{2}}{4}\right) \operatorname{erf}\left(\frac{\mathrm{i} \sqrt{\theta}}{2} s\right) \\
&-\mathrm{i} \sqrt{\theta \pi} s \exp \left(-s-\frac{\theta s^{2}}{4}\right) \operatorname{erf}\left(\frac{\mathrm{i} \sqrt{\theta}}{2} s\right) \\
&+\frac{\mathrm{i} \sqrt{\theta \pi}}{2} \exp \left(-s-\frac{\theta s^{2}}{4}\right) \operatorname{erf}\left(\frac{\mathrm{i} \sqrt{\theta}}{2} s\right) \\
&-\mathrm{i} \sqrt{\pi / \theta} s^{2} \exp \left(-s-\frac{\theta s^{2}}{4}\right) \operatorname{erf}\left(\frac{\mathrm{i} \sqrt{\theta}}{2} s\right)-\frac{\theta}{2} s \exp (-s), \\
& g_{X, S}(s)=-\frac{\mathrm{i} \sqrt{\pi / \theta}}{8} s^{3} \exp \left(-s-\frac{\theta s^{2}}{4}\right) \operatorname{erf}\left(\frac{\mathrm{i} \sqrt{\theta}}{2} s\right) \\
&-\frac{\mathrm{i} \sqrt{\theta \pi}}{2} s^{2} \exp \left(-s-\frac{\theta s^{2}}{4}\right) \operatorname{erf}\left(\frac{\mathrm{i} \sqrt{\theta}}{2} s\right)
\end{aligned}
$$




$$
\begin{aligned}
& +\frac{\mathrm{i} \sqrt{\theta \pi}}{4} s \exp \left(-s-\frac{\theta s^{2}}{4}\right) \operatorname{erf}\left(\frac{\mathrm{i} \sqrt{\theta}}{2} s\right) \\
& -\frac{\mathrm{i} \sqrt{\pi}}{2} s \exp \left(-s-\frac{\theta s^{2}}{4}\right) \operatorname{erf}\left(\frac{\mathrm{i} \sqrt{\theta}}{2} s\right)-\frac{\theta}{4} s \exp (-s)
\end{aligned}
$$

for $0<\theta<1, x>0$ and $y>0$, where $\mathrm{i}=\sqrt{-1}$.

\section{Lawrance and Lewis' (1980) bivariate exponential distribution:}

$$
\begin{aligned}
& f(x, y)=I\{\beta y<x<y / \beta\} \frac{1}{1+\beta} \exp \left(-\frac{x+y}{1+\beta}\right)+\frac{1}{\beta} \exp \left(-\frac{x+y}{\beta}\right), \\
& f_{S}(s)=\frac{(1-\beta) s}{(1+\beta)^{2}} \exp \left(-\frac{s}{1+\beta}\right)+\frac{s}{\beta} \exp \left(-\frac{s}{\beta}\right), \\
& F_{S}(s)=\frac{1-\beta}{(1+\beta)^{2}}\left[(1+\beta)^{2}-(1+\beta)^{2} \exp \left(-\frac{s}{1+\beta}\right)-(1+\beta) s \exp \left(-\frac{s}{1+\beta}\right)\right] \\
& +\frac{1}{\beta}\left[\beta^{2}-\beta^{2} \exp \left(-\frac{s}{\beta}\right)-\beta s \exp \left(-\frac{s}{\beta}\right)\right] \text {, } \\
& E\left[S 1_{\{S>b\}}\right]=\frac{(1-\beta)(1+\beta+b)}{1+\beta} \exp \left(-\frac{b}{1+\beta}\right)+(\beta+b) \exp \left(-\frac{b}{\beta}\right), \\
& g_{X, S}(s)=\frac{\left(1-\beta^{2}\right) s^{2}}{2(1+\beta)^{3}} \exp \left(-\frac{s}{1+\beta}\right)+\frac{s^{2}}{2 \beta} \exp \left(-\frac{s}{\beta}\right) \text {, } \\
& E\left[X 1_{\{S>b\}}\right]=\left(1-\beta^{2}\right)\left[1+\frac{b}{1+\beta}+\frac{b^{2}}{2(1+\beta)^{2}}\right] \exp \left(-\frac{b}{1+\beta}\right) \\
& +\left(\beta^{2}+b \beta+\frac{b^{2}}{2}\right) \exp \left(-\frac{b}{\beta}\right)
\end{aligned}
$$

for $0<\beta \leq 1, x>0$ and $y>0$.

\section{Block and Basu (1976)'s bivariate exponential distribution:}

$$
\begin{aligned}
& f(x, y)= \begin{cases}C \exp (-\alpha x-\beta y), & \text { if } x>y, \\
C \exp (-\gamma x-\delta y), & \text { if } x \leq y,\end{cases} \\
& f_{S}(s)= \frac{C}{\beta-\alpha}\left[\exp (-\alpha s)-\exp \left(-\frac{(\alpha+\beta) s}{2}\right)\right]-\frac{D}{\delta-\gamma}\left[\exp (-\delta s)-\exp \left(-\frac{(\delta+\gamma) s}{2}\right)\right], \\
& F_{S}(s)= \frac{C}{\beta-\alpha}\left[\frac{1-\exp (-\alpha s)}{\alpha}-\frac{2}{\alpha+\beta}\left(1-\exp \left(-\frac{(\alpha+\beta) s}{2}\right)\right)\right] \\
&-\frac{D}{\delta-\gamma}\left[\frac{2}{\delta+\gamma}\left(1-\exp \left(-\frac{(\delta+\gamma s}{2}\right)\right)-\frac{1-\exp (-\delta s)}{\delta}\right] \\
& E\left[S 1_{\{S>b\}}\right]=\frac{C}{\beta-\alpha}\left[\frac{(b \alpha+1) \exp (-b \alpha)}{\alpha^{2}}-\frac{4}{(\alpha+\beta)^{2}}\left(b \frac{\alpha+\beta}{2}+1\right) \exp \left(-b \frac{\alpha+\beta}{2}\right)\right] \\
&-\frac{D}{\delta-\gamma}\left[\frac{4}{(\delta+\gamma)^{2}}\left(b \frac{\delta+\gamma}{2}+1\right) \exp \left(-b \frac{\delta+\gamma}{2}\right)-\frac{(b \delta+1) \exp (-b \delta)}{\delta^{2}}\right] \\
& g_{X, S}(s)= C\left[\frac{\exp (-\beta s)-\exp (-\alpha s)}{(\alpha-\beta)^{2}}-\frac{s \exp (-\alpha s)}{\alpha-\beta}\right] \\
&+D\left[\frac{\exp (-\delta s)-\exp (-(\delta+\gamma) s / 2)}{(\delta-\gamma)^{2}}-\frac{s \exp (-(\delta+\gamma) s / 2)}{2(\delta-\gamma)}\right]
\end{aligned}
$$




$$
\begin{aligned}
-C & {\left[\frac{\exp (-\beta s)-\exp (-\beta s-(\gamma-\delta) s / 2)}{(\delta-\gamma)^{2}}-\frac{s \exp (-\beta s-(\gamma-\delta) s / 2)}{2(\delta-\gamma)}\right], } \\
E\left[X 1_{\{S>b\}}\right]=C\left[-\frac{(b \alpha+1) \exp (-b \alpha)}{\alpha^{2}(\alpha-\beta)}+\frac{\exp (-b \beta)}{\beta(\alpha-\beta)^{2}}-\frac{\exp (-b \alpha)}{\alpha(\alpha-\beta)^{2}}\right] & \\
+D & {\left[\frac{\exp (-b \delta)}{\delta(\delta-\gamma)^{2}}-\frac{2 \exp (-(\delta+\gamma) b / 2)}{(\delta-\gamma)^{2}(\delta+\gamma)}-\frac{(2+(\delta+\gamma) b) \exp (-(\delta+\gamma) b / 2)}{(\delta+\gamma)^{2}(\delta-\gamma)}\right] } \\
-C\left[\frac{\exp (-b \beta)}{\beta(\delta-\gamma)^{2}}-\frac{\exp (-b \beta-(\gamma-\delta) b / 2)}{(\delta-\gamma)^{2}(\beta+(\gamma-\delta) / 2)}\right] & \\
+C & \frac{(2+2 b \beta+b(\gamma-\delta)) \exp (-b \beta-(\gamma-\delta) b / 2)}{(\delta-\gamma)(2 \beta+\gamma-\delta)^{2}}
\end{aligned}
$$

for $\alpha>0, \beta>0, \gamma>0, \delta>0, x>0$ and $y>0$.

Arnold and Strauss (1991)'s bivariate exponential distribution:

$$
\begin{aligned}
f(x, y)= & C \exp (-\gamma x-\delta y-\theta x y) \\
f_{S}(s)= & C \sqrt{\pi} p^{-1 / 2} \exp \left(-\delta s+\frac{q^{2}}{4 p}\right)\left[\Phi\left(\sqrt{2 p} s-(2 p)^{-1 / 2} q\right)-\Phi\left(-(2 p)^{-1 / 2} q\right)\right] \\
g_{X, S}(s)= & 2^{-1} C \sqrt{\pi} p^{-3 / 2} q \exp \left(-\delta s+\frac{q^{2}}{4 p}\right)\left[\Phi\left(\sqrt{2 p} s-(2 p)^{-1 / 2} q\right)-\Phi\left(-(2 p)^{-1 / 2} q\right)\right] \\
& -2^{-1} C p^{-1} \exp \left(-\delta s+\frac{q^{2}}{4 p}\right)\left[\exp \left(-p s^{2}+q s\right)-1\right]
\end{aligned}
$$

for $-\infty<\gamma<\infty,-\infty<\delta<\infty,-\infty<\theta<\infty, x>0$ and $y>0$, where $p=-\theta$ and $q=\delta-\theta s$.

Beta exponential distribution in equation (8.41) of Balakrishnan and Cai (2009):

$$
\begin{aligned}
& f(x, y)=C x^{p-1}(y-x)^{q-1} \exp (-a y), \\
& f_{S}(s)=C 2^{-p} B(p, q) \exp (-a s) s^{p+q-1}{ }_{1} F_{1}\left(p ; p+q ; \frac{a s}{2}\right), \\
& F_{S}(s)=C 2^{-p} B(p, q) \sum_{k=0}^{\infty} \frac{(p)_{k} a^{k} \gamma(p+q+k, a s)}{(p+q)_{k} k ! 2^{k} a^{p+q+k}}, \\
& E\left[S 1_{\{S>b\}}\right]=C 2^{-p} B(p, q) \frac{(p)_{k} a^{k} \Gamma(p+q+k+1, a b)}{(p+q)_{k} k ! 2^{k} a^{p+q+k+1}}, \\
& g_{X, S}(s)=C 2^{-p-1} B(p+1, q) \exp (-a s) s^{p+q}{ }_{1} F_{1}\left(p+1 ; p+q+1 ; \frac{a s}{2}\right), \\
& E\left[X 1_{\{S>b\}}\right]=C 2^{-p-1} B(p+1, q) \frac{(p+1)_{k} a^{k} \Gamma(p+q+k+2, a b)}{(p+q+1)_{k} k ! 2^{k} a^{p+q+k+1}}
\end{aligned}
$$

for $a>0, p>0, q>0$, and $y>x>0$.

Mixtures of independent gamma distributions with integer shape parameters:

$$
\begin{aligned}
& f(x, y)=\sum_{k=0}^{\infty} C_{k} x^{\alpha_{k}-1} y^{\beta_{k}-1} \exp \left(-\gamma_{k} x-\delta_{k} y\right), \\
& f_{S}(s)=\sum_{k=0}^{\infty} C_{k} B\left(\alpha_{k}, \beta_{k}\right) \exp \left(-\delta_{k} s\right){ }_{1} F_{1}\left(\alpha_{k} ; \alpha_{k}+\beta_{k} ;\left(\delta_{k}-\gamma_{k}\right) s\right),
\end{aligned}
$$




$$
\begin{aligned}
& F_{S}(s)= \sum_{k=0}^{\infty} \sum_{i=0}^{\beta_{k}-1} C_{k}\left(\begin{array}{c}
\beta_{k}-1 \\
i
\end{array}\right)(-1)^{i} \frac{\left(\alpha_{k}+i-1\right) ! \gamma\left(\beta_{k}-i, \delta_{k} s\right)}{\left(\gamma_{k}-\delta_{k}\right)^{\alpha_{k}+i} \delta_{k}^{\beta_{k}-i}} \\
&-\sum_{k=0}^{\infty} \sum_{i=0}^{\beta_{k}-1} \sum_{j=0}^{\alpha_{k}+i-1} C_{k}\left(\begin{array}{c}
\beta_{k}-1 \\
i
\end{array}\right)(-1)^{i} \frac{\left(\alpha_{k}+i-1\right) !\left(\gamma_{k}-\delta_{k}\right)^{j} \gamma\left(\beta_{k}-i+j, \delta_{k} s\right)}{\left(\gamma_{k}-\delta_{k}\right)^{\alpha_{k}+i} j ! \gamma_{k}^{\beta_{k}-i+j}}, \\
& E\left[S 1_{\{S>b\}}\right]=\sum_{k=0}^{\infty} \sum_{i=0}^{\beta_{k}-1} C_{k}\left(\begin{array}{c}
\beta_{k}-1 \\
i
\end{array}\right)(-1)^{i} \frac{\left(\alpha_{k}+i-1\right) ! \Gamma\left(\beta_{k}-i+1, \delta_{k} b\right)}{\left(\gamma_{k}-\delta_{k}\right)^{\alpha_{k}+i} \delta_{k}^{\beta_{k}-i+1}} \\
&-\sum_{k=0}^{\infty} \sum_{i=0}^{\beta_{k}-1} \sum_{j=0}^{\alpha_{k}+i-1} C_{k}\left(\begin{array}{c}
\beta_{k}-1 \\
i
\end{array}\right)(-1)^{i} \frac{\left(\alpha_{k}+i-1\right) !\left(\gamma_{k}-\delta_{k}\right)^{j} \gamma\left(\beta_{k}-i+j+1, \delta_{k} b\right)}{\left(\gamma_{k}-\delta_{k}\right)^{\alpha_{k}+i} j ! \gamma_{k}^{\beta_{k}-i+j+1}}, \\
& g_{X, S}(s)= \sum_{k=0}^{\infty} \sum_{i=0}^{\beta_{k}-1} C_{k}\left(\begin{array}{c}
\beta_{k}-1 \\
i
\end{array}\right)(-1)^{i} \frac{\left(\alpha_{k}+i\right) ! s^{\beta_{k}-1-i} \exp \left(-\delta_{k} s\right)}{\left(\gamma_{k}-\delta_{k}\right)^{\alpha_{k}+i+1}} \\
&-\sum_{k=0}^{\infty} \sum_{i=0}^{\beta_{k}-1} \sum_{j=0}^{\alpha_{k}+i} C_{k}\left(\begin{array}{c}
\beta_{k}-1 \\
i
\end{array}\right)(-1)^{i} \frac{\left(\alpha_{k}+i\right) !}{(\gamma-\delta)^{\alpha_{k}+i+1-j} j !}, \\
& E\left[X 1_{\{S>b\}}\right]=\sum_{k=0}^{\infty} \sum_{i=0}^{\beta_{k}-1} C_{k}\left(\begin{array}{c}
\beta_{k}-1 \\
i
\end{array}\right)(-1)^{i} \frac{\left(\alpha_{k}+i\right) ! \Gamma\left(\beta_{k}-i, \delta_{k} b\right)}{\left(\gamma_{k}-\delta_{k}\right)^{\alpha_{k}+i+1} b^{\beta_{k}-i}} \\
&-\sum_{k=0}^{\infty} \sum_{i=0}^{\beta_{k}-1} \sum_{j=0}^{\alpha_{k}+i} C_{k}\left(\begin{array}{c}
\beta_{k}-1 \\
i
\end{array}\right)(-1)^{i} \frac{\left(\alpha_{k}+i\right) ! \Gamma\left(\beta_{k}-i+j, \delta_{k} b\right)}{\left(\gamma_{k}-\delta_{k}\right)^{\alpha_{k}+i-j+1} j ! b^{\beta_{k}-i+j}}
\end{aligned}
$$

for $\alpha_{k}>0, \beta_{k}>0, \gamma_{k}>0, \delta_{k}>0, x>0$ and $y>0$, provided that $\alpha_{k}$ and $\beta_{k}$ are integers.

\section{Mixtures of independent gamma distributions with real shape parameters:}

$$
\begin{aligned}
& f(x, y)=\sum_{k=0}^{\infty} C_{k} x^{\alpha_{k}-1} y^{\beta_{k}-1} \exp \left(-\gamma_{k} x-\delta_{k} y\right), \\
& f_{S}(s)=\sum_{k=0}^{\infty} C_{k} B\left(\alpha_{k}, \beta_{k}\right) \exp \left(-\delta_{k} s\right){ }_{1} F_{1}\left(\alpha_{k} ; \alpha_{k}+\beta_{k} ;\left(\delta_{k}-\gamma_{k}\right) s\right), \\
& F_{S}(s)=\sum_{k=0}^{\infty} \sum_{i=0}^{\infty} C_{k} B\left(\alpha_{k}, \beta_{k}\right) \frac{\left(\alpha_{k}\right)_{i}\left(\delta_{k}-\gamma_{k}\right)^{i} \gamma\left(i+\alpha_{k}+\beta_{k}, \delta_{k} s\right)}{\left(\alpha_{k}+\beta_{k}\right)_{i} \delta_{k}^{\alpha_{k}+\beta_{k}+i}}, \\
& E\left[S 1_{\{S>b\}}\right]=\sum_{k=0}^{\infty} \sum_{i=0}^{\infty} C_{k} B\left(\alpha_{k}, \beta_{k}\right) \frac{\left(\alpha_{k}\right)_{i}\left(\delta_{k}-\gamma_{k}\right)^{i} \Gamma\left(i+\alpha_{k}+\beta_{k}+1, \delta_{k} b\right)}{\left(\alpha_{k}+\beta_{k}\right)_{i} \delta_{k}^{\alpha_{k}+\beta_{k}+i+1}}, \\
& g_{X, S}(s)=\sum_{k=0}^{\infty} \sum_{i=0}^{\infty} C_{k} B\left(\alpha_{k}+1, \beta_{k}\right) \frac{\left(\alpha_{k}+1\right)_{i}\left(\delta_{k}-\gamma_{k}\right)^{i} s^{i+\alpha_{k}+\beta_{k}} \exp \left(-\delta_{k} s\right)}{\left(1+\alpha_{k}+\beta_{k}\right)_{i} i !}, \\
& E\left[X 1_{\{S>b\}}\right]=\sum_{k=0}^{\infty} \sum_{i=0}^{\infty} C_{k} B\left(\alpha_{k}+1, \beta_{k}\right) \frac{\left(\alpha_{k}+1\right)_{i}\left(\delta_{k}-\gamma_{k}\right)^{i} \Gamma\left(i+\alpha_{k}+\beta_{k}+1, \delta_{k} b\right)}{\left(1+\alpha_{k}+\beta_{k}\right)_{i} i ! \delta_{k}^{\alpha_{k}+\beta_{k}+i+1}}
\end{aligned}
$$

for $\alpha_{k}>0, \beta_{k}>0, \gamma_{k}>0, \delta_{k}>0, x>0$ and $y>0$.

Nadarajah and Gupta (2006)'s bivariate gamma distribution with equal scale param- 
eters:

$$
\begin{aligned}
& f(x, y)=\sum_{k=0}^{\infty} C_{k} x^{\alpha_{k}-1} y^{\beta_{k}-1}(x+y)^{\gamma_{k}} \exp \left(-p_{k} x-p_{k} y\right) \\
& f_{S}(s)=\sum_{k=0}^{\infty} C_{k} B\left(\alpha_{k}, \beta_{k}\right) s^{\alpha_{k}+\beta_{k}+\gamma_{k}-1} \exp \left(-p_{k} s\right) \\
& F_{S}(s)=\sum_{k=0}^{\infty} C_{k} B\left(\alpha_{k}, \beta_{k}\right) p_{k}^{-\alpha_{k}-\beta_{k}-\gamma_{k}} \gamma\left(\alpha_{k}+\beta_{k}+\gamma_{k}, p_{k} s\right) \\
& g_{X, S}(s)=\sum_{k=0}^{\infty} C_{k} B\left(\alpha_{k}+1, \beta_{k}\right) s^{\alpha_{k}+\beta_{k}+\gamma_{k}} \exp \left(-p_{k} s\right), \\
& E\left[S 1_{\{S>b\}}\right]=\sum_{k=0}^{\infty} C_{k} B\left(\alpha_{k}, \beta_{k}\right) p_{k}^{-\alpha_{k}-\beta_{k}-\gamma_{k}-1} \Gamma\left(\alpha_{k}+\beta_{k}+\gamma_{k}+1, p_{k} b\right), \\
& E\left[X 1_{\{S>b\}}\right]=\sum_{k=0}^{\infty} C_{k} B\left(\alpha_{k}+1, \beta_{k}\right) p_{k}^{-\alpha_{k}-\beta_{k}-\gamma_{k}-1} \Gamma\left(\alpha_{k}+\beta_{k}+\gamma_{k}+1, p_{k} b\right)
\end{aligned}
$$

for $\alpha_{k}>0, \beta_{k}>0, \gamma_{k}>0, p_{k}>0, x>0$ and $y>0$.

Nadarajah and Gupta (2006)'s bivariate gamma distribution with unequal scale parameters:

$$
\begin{aligned}
f(x, y)= & \sum_{k=0}^{\infty} C_{k} x^{\alpha_{k}-1} y^{\beta_{k}-1}\left(p_{k} x+q_{k} y\right)^{\gamma_{k}} \exp \left(-p_{k} x-q_{k} y\right), \\
f_{S}(s)= & \sum_{k=0}^{\infty} C_{k} q_{k}^{\gamma_{k}} \exp \left(-q_{k} s\right) \sum_{i=0}^{\infty}\left(\begin{array}{c}
\gamma_{k} \\
i
\end{array}\right)\left(\frac{p_{k}}{q_{k}}-1\right)^{i} B\left(i+\alpha_{k}, \beta_{k}\right) s^{\alpha_{k}+\beta_{k}+\gamma_{k}-1} \\
& { }_{1} F_{1}\left(i+\alpha_{k} ; i+\alpha_{k}+\beta_{k} ;-\left(p_{k}-q_{k}\right) s\right), \\
F_{S}(s)= & \sum_{k=0}^{\infty} C_{k} q_{k}^{\gamma_{k}} \exp \left(-q_{k} s\right) \sum_{i=0}^{\infty}\left(\begin{array}{c}
\gamma_{k} \\
i
\end{array}\right)\left(\frac{p_{k}}{q_{k}}-1\right)^{i} B\left(i+\alpha_{k}, \beta_{k}\right) \\
& \cdot \sum_{j=0}^{\infty} \frac{\left(i+\alpha_{k}\right)_{j}(-1)^{j}\left(p_{k}-q_{k}\right)^{i}}{\left(i+\alpha_{k}+\beta_{k}\right)_{j} j !} \frac{s^{\alpha_{k}+\beta_{k}+\gamma_{k}+j}}{\alpha_{k}+\beta_{k}+\gamma_{k}+j}, \\
g_{X, S}(s)= & \sum_{k=0}^{\infty} C_{k} q_{k}^{\gamma_{k}} \exp \left(-q_{k} s\right) \sum_{i=0}^{\infty}\left(\begin{array}{c}
\gamma_{k} \\
i
\end{array}\right)\left(\frac{p_{k}}{q_{k}}-1\right)^{i} B\left(i+\alpha_{k}+1, \beta_{k}\right) s^{\alpha_{k}+\beta_{k}+\gamma_{k}} \\
& \cdot 1 F_{1}\left(i+\alpha_{k}+1 ; i+\alpha_{k}+\beta_{k}+1 ;-\left(p_{k}-q_{k}\right) s\right)
\end{aligned}
$$

for $\alpha_{k}>0, \beta_{k}>0, \gamma_{k}>0, p_{k}>0, q_{k}>0, x>0$ and $y>0$.

Nagar and Sepulveda-Murillo (2011)'s bivariate confluent hypergeometric distribution:

$$
\begin{aligned}
& f(x, y)=C x^{p-1} y^{q-1}{ }_{1} F_{1}(\alpha ; \beta ;-x-y), \\
& f_{S}(s)=C B(p, q) s^{p+q-1}{ }_{1} F_{1}(\alpha ; \beta ;-s), \\
& F_{S}(s)=C B(p, q) \sum_{k=0}^{\infty} \frac{(\alpha)_{k}(-1)^{k}}{(\beta)_{k} k !} \frac{s^{p+q+k}}{p+q+k}, \\
& g_{X, S}(s)=C B(p+1, q) s^{p+q}{ }_{1} F_{1}(\alpha ; \beta ;-s)
\end{aligned}
$$


for $p>0, q>0, \alpha>0, \beta>0, x>0$ and $y>0$.

Becker and Roux (1981)'s bivariate gamma distribution:

$$
\begin{aligned}
f(x, y)= & \begin{cases}C x^{\alpha-1}(x+p y)^{\beta-1} \exp (-\gamma x-\delta y), \quad \text { if } x<y, \\
C x^{c-1}(x+q y)^{d-1} \exp (-e x-f y), \quad \text { if } x<y,\end{cases} \\
f_{S}(s)= & 2^{-\alpha} \alpha^{-1} C s^{\alpha+\beta-1} \exp (-\delta s) \Phi_{1}\left(\alpha, 1-\beta, 1+\alpha,(\delta-\gamma) \frac{s}{2},-\frac{1-p}{2 p}\right) \\
& +q^{d-1} c^{-1} D s^{c+d-1} \exp (-f s) \Phi_{1}\left(c, 1-d, 1+c,(f-e) s, \frac{q-1}{q}\right)
\end{aligned}
$$

for $\alpha>0, \beta>0, \gamma>0, \delta>0, c>0, d>0, e>0, f>0, p>0, q>0, x>0$ and $y>0$.

Mohsin et al. (2013)'s bivariate gamma distribution:

$$
\begin{aligned}
& f(x, y)=\frac{\beta^{\alpha} \delta^{\gamma}}{\Gamma(\alpha) \Gamma(\gamma)} x^{\alpha-\gamma-1} y^{\gamma-1} \exp \left(-\beta x-\frac{\delta y}{x}\right) \\
& f_{S}(s)=\frac{\beta^{\alpha} \delta^{\gamma}}{\Gamma(\alpha)} \sum_{k=1}^{\infty} \frac{(-\beta)^{k}}{k !} s^{\alpha+k-1} U(\delta, \gamma, \gamma-\alpha-k+1), \\
& g_{X, S}(s)=\frac{\beta^{\alpha} \delta^{\gamma}}{\Gamma(\alpha)} \sum_{k=1}^{\infty} \frac{(-\beta)^{k}}{k !} s^{\alpha+k} U(\delta, \gamma, \gamma-\alpha-k)
\end{aligned}
$$

for $\alpha>0, \beta>0, \gamma>0, \delta>0, x>0$ and $y>0$.

Cheriyan (1941)'s bivariate gamma distribution in equation (8.31) of Balakrishnan and Cai (2009):

$$
\begin{aligned}
& f(x, y)=C \exp (-x-y) \int_{0}^{\min (x, y)}(x-z)^{\theta_{1}}(y-z)^{\theta_{2}-1} z^{\theta_{2}-1} \exp (z) d z, \\
& f_{S}(s)=C B\left(\theta_{1}+1, \theta_{2}\right) \exp (-s) \\
& \cdot \int_{0}^{s / 2} x^{\theta_{1}+\theta_{3}}(s-x)^{\theta_{2}-1} \Phi_{1}\left(\theta_{3}, 1-\theta_{2}, \theta_{1}+\theta_{3}+1, x, \frac{x}{1-x}\right) d x \\
& +C B\left(\theta_{2}, \theta_{3}\right) \exp (-s) \\
& \cdot \int_{s / 2}^{1} x^{\theta_{1}}(s-x)^{\theta_{2}+\theta_{3}-1} \Phi_{1}\left(\theta_{3},-\theta_{1}, \theta_{2}+\theta_{3}, s-x, \frac{s-x}{x}\right) d x \\
& g_{X, S}(s)=C B\left(\theta_{1}+1, \theta_{2}\right) \exp (-s) \\
& \cdot \int_{0}^{s / 2} x^{\theta_{1}+\theta_{3}+1}(s-x)^{\theta_{2}-1} \Phi_{1}\left(\theta_{3}, 1-\theta_{2}, \theta_{1}+\theta_{3}+1, x, \frac{x}{1-x}\right) d x \\
& +C B\left(\theta_{2}, \theta_{3}\right) \exp (-s)
\end{aligned}
$$




$$
\cdot \int_{s / 2}^{1} x^{\theta_{1}+1}(s-x)^{\theta_{2}+\theta_{3}-1} \Phi_{1}\left(\theta_{3},-\theta_{1}, \theta_{2}+\theta_{3}, s-x, \frac{s-x}{x}\right) d x
$$

for $\theta_{1}>0, \theta_{2}>0, \theta_{3}>0, x>0$ and $y>0$.

Dussauchoy and Berland (1975)'s bivariate gamma distribution:

$$
\begin{aligned}
& f(x, y)=\sum_{k=0}^{\infty} C_{k} x^{\alpha_{k}-1}(y-\beta x)^{\theta_{k}-1} \exp \left[-\gamma_{k} x-\delta_{k}(y-\beta x)\right] \\
& f_{S}(s)=\sum_{k=0}^{\infty} \frac{C_{k} s^{\theta_{k}-1} \exp \left(-\delta_{k} s\right)}{\alpha_{k}(1+\beta)^{\alpha_{k}}} \Phi_{1}\left(\alpha_{k}, 1-\theta_{k}, 1+\alpha_{k}, \delta_{k}-\frac{\gamma_{k}}{1+\beta}, \frac{1}{s}\right), \\
& g_{X, S}(s)=\sum_{k=0}^{\infty} \frac{C_{k} s^{\theta_{k}-1} \exp \left(-\delta_{k} s\right)}{\left(\alpha_{k}+1\right)(1+\beta)^{\alpha_{k}+1}} \Phi_{1}\left(\alpha_{k}+1,1-\theta_{k}, 1+\alpha_{k}, \delta_{k}-\frac{\gamma_{k}}{1+\beta}, \frac{1}{s}\right)
\end{aligned}
$$

for $\alpha_{k}>0, \beta>0, \gamma_{k}>0, \delta_{k}>0$ and $y>\beta x>0$.

\section{Mixtures of independent two piece gamma distributions:}

$$
\begin{aligned}
& f(x, y)= \begin{cases}\sum_{k=1}^{\infty} C_{k} x^{\alpha_{k}-1} y^{\beta_{k}-1} \exp \left(-\gamma_{k} x-\delta_{k} y\right), & \text { if } x<y, \\
\sum_{k=1}^{\infty} D_{k} x^{p_{k}-1} y^{q_{k}-1} \exp \left(-r_{k} x-t_{k} y\right), & \text { if } x \geq y,\end{cases} \\
& f_{S}(s)=\sum_{k=1}^{\infty} \sum_{i=0}^{\beta_{k}-1} C_{k}\left(\begin{array}{c}
\beta_{k}-1 \\
i
\end{array}\right) \frac{(-1)^{i}\left(\alpha_{k}+i-1\right) !}{\left(\delta_{k}-\gamma_{k}\right)^{\alpha_{k}+i}} s^{\beta_{k}-i-1} \exp \left(-\delta_{k} s\right) \\
& -\sum_{k=1}^{\infty} \sum_{i=0}^{\beta_{k}-1} \sum_{j=0}^{\alpha_{k}+i-1} C_{k}\left(\begin{array}{c}
\beta_{k}-1 \\
i
\end{array}\right) \frac{(-1)^{i}\left(\alpha_{k}+i-1\right) !}{\left(\delta_{k}-\gamma_{k}\right)^{\alpha_{k}+i-j} 2^{j} j !} s^{\beta_{k}-i+j-1} \exp \left(-\frac{\gamma_{k} s}{2}-\frac{\delta_{k} s}{2}\right) \\
& +\sum_{k=1}^{\infty} \sum_{i=0}^{q_{k}-1} \sum_{j=0}^{p_{k}+i-1} D_{k}\left(\begin{array}{c}
q_{k}-1 \\
i
\end{array}\right) \frac{(-1)^{i}\left(p_{k}+i-1\right) !}{\left(t_{k}-r_{k}\right)^{p_{k}+i-j} 2^{j} j !} s^{q_{k}-i+j-1} \exp \left(-\frac{r_{k} s}{2}-\frac{t_{k} s}{2}\right) \\
& -\sum_{k=1}^{\infty} \sum_{i=0}^{q_{k}-1} \sum_{j=0}^{p_{k}+i-1} D_{k}\left(\begin{array}{c}
q_{k}-1 \\
i
\end{array}\right) \frac{(-1)^{i}\left(p_{k}+i-1\right) !}{\left(t_{k}-r_{k}\right)^{p_{k}+i-j} j !} s^{q_{k}-i+j-1} \exp \left(-r_{k} s\right), \\
& F_{S}(s)=\sum_{k=1}^{\infty} \sum_{i=0}^{\beta_{k}-1} C_{k}\left(\begin{array}{c}
\beta_{k}-1 \\
i
\end{array}\right) \frac{(-1)^{i}\left(\alpha_{k}+i-1\right) ! \gamma\left(\beta_{k}-i, \delta_{k} s\right)}{\left(\delta_{k}-\gamma_{k}\right)^{\alpha_{k}+i} \delta_{k}^{\beta_{k}-i}} \\
& -\sum_{k=1}^{\infty} \sum_{i=0}^{\beta_{k}-1} \sum_{j=0}^{\alpha_{k}+i-1} C_{k}\left(\begin{array}{c}
\beta_{k}-1 \\
i
\end{array}\right) \frac{(-1)^{i}\left(\alpha_{k}+i-1\right) ! 2^{\beta_{k}-i} \gamma\left(\beta_{k}-i+j, \frac{\left(\gamma_{k}+\delta_{k}\right) s}{2}\right)}{\left(\delta_{k}-\gamma_{k}\right)^{\alpha_{k}+i-j} j !\left(\gamma_{k}+\delta_{k}\right)^{\beta_{k}-i+j}} \\
& +\sum_{k=1}^{\infty} \sum_{i=0}^{q_{k}-1} \sum_{j=0}^{p_{k}+i-1} D_{k}\left(\begin{array}{c}
q_{k}-1 \\
i
\end{array}\right) \frac{(-1)^{i}\left(p_{k}+i-1\right) ! 2^{q_{k}-i} \gamma\left(q_{k}-i+j, \frac{\left(t_{k}+r_{k}\right) s}{2}\right)}{\left(t_{k}-r_{k}\right)^{p_{k}+i-j}\left(t_{k}+r_{k}\right)^{q_{k}-i+j} j !} \\
& -\sum_{k=1}^{\infty} \sum_{i=0}^{q_{k}-1} \sum_{j=0}^{p_{k}+i-1} D_{k}\left(\begin{array}{c}
q_{k}-1 \\
i
\end{array}\right) \frac{(-1)^{i}\left(p_{k}+i-1\right) ! \gamma\left(q_{k}-i+j, r_{k} s\right)}{\left(t_{k}-r_{k}\right)^{p_{k}+i-j} r_{k}^{q_{k}-i+j} j !} \\
& E\left[S 1_{\{S>b\}}\right]=\sum_{k=1}^{\infty} \sum_{i=0}^{\beta_{k}-1} C_{k}\left(\begin{array}{c}
\beta_{k}-1 \\
i
\end{array}\right) \frac{(-1)^{i}\left(\alpha_{k}+i-1\right) ! \Gamma\left(\beta_{k}-i+1, \delta_{k} b\right)}{\left(\delta_{k}-\gamma_{k}\right)^{\alpha_{k}+i} \delta_{k}^{\beta_{k}-i+1}}
\end{aligned}
$$




$$
\begin{aligned}
& -\sum_{k=1}^{\infty} \sum_{i=0}^{\beta_{k}-1} \sum_{j=0}^{\alpha_{k}+i-1} C_{k}\left(\begin{array}{c}
\beta_{k}-1 \\
i
\end{array}\right) \frac{(-1)^{i}\left(\alpha_{k}+i-1\right) ! 2^{\beta_{k}-i} \Gamma\left(\beta_{k}-i+j+1, \frac{\left(\gamma_{k}+\delta_{k}\right) b}{2}\right)}{\left(\delta_{k}-\gamma_{k}\right)^{\alpha_{k}+i-j} j !\left(\gamma_{k}+\delta_{k}\right)^{\beta_{k}-i+j+1}} \\
& +\sum_{k=1}^{\infty} \sum_{i=0}^{q_{k}-1} \sum_{j=0}^{p_{k}+i-1} D_{k}\left(\begin{array}{c}
q_{k}-1 \\
i
\end{array}\right) \frac{(-1)^{i}\left(p_{k}+i-1\right) ! 2^{q_{k}-i} \Gamma\left(q_{k}-i+j+1, \frac{\left(t_{k}+r_{k}\right) b}{2}\right)}{\left(t_{k}-r_{k}\right)^{p_{k}+i-j}\left(t_{k}+r_{k}\right)^{q_{k}-i+j+1} j !} \\
& -\sum_{k=1}^{\infty} \sum_{i=0}^{q_{k}-1} \sum_{j=0}^{p_{k}+i-1} D_{k}\left(\begin{array}{c}
q_{k}-1 \\
i
\end{array}\right) \frac{(-1)^{i}\left(p_{k}+i-1\right) ! \Gamma\left(q_{k}-i+j+1, r_{k} b\right)}{\left(t_{k}-r_{k}\right)^{p_{k}+i-j} r_{k}^{q_{k}-i+j+1} j !} \\
& g_{X, S}(s)=\sum_{k=1}^{\infty} \sum_{i=0}^{\beta_{k}-1} C_{k}\left(\begin{array}{c}
\beta_{k}-1 \\
i
\end{array}\right) \frac{(-1)^{i}\left(\alpha_{k}+i\right) !}{\left(\delta_{k}-\gamma_{k}\right)^{\alpha_{k}+i+1}} s^{\beta_{k}-i-1} \exp \left(-\delta_{k} s\right) \\
& -\sum_{k=1}^{\infty} \sum_{i=0}^{\beta_{k}-1} \sum_{j=0}^{\alpha_{k}+i} C_{k}\left(\begin{array}{c}
\beta_{k}-1 \\
i
\end{array}\right) \frac{(-1)^{i}\left(\alpha_{k}+i\right) !}{\left(\delta_{k}-\gamma_{k}\right)^{\alpha_{k}+i-j+1} 2^{j} j !} s^{\beta_{k}-i+j-1} \exp \left(-\frac{\gamma_{k} s}{2}-\frac{\delta_{k} s}{2}\right) \\
& +\sum_{k=1}^{\infty} \sum_{i=0}^{q_{k}-1} \sum_{j=0}^{p_{k}+i} D_{k}\left(\begin{array}{c}
q_{k}-1 \\
i
\end{array}\right) \frac{(-1)^{i}\left(p_{k}+i\right) !}{\left(t_{k}-r_{k}\right)^{p_{k}+i-j+1} 2^{j} j !} s^{q_{k}-i+j-1} \exp \left(-\frac{r_{k} s}{2}-\frac{t_{k} s}{2}\right) \\
& -\sum_{k=1}^{\infty} \sum_{i=0}^{q_{k}-1} \sum_{j=0}^{p_{k}+i} D_{k}\left(\begin{array}{c}
q_{k}-1 \\
i
\end{array}\right) \frac{(-1)^{i}\left(p_{k}+i\right) !}{\left(t_{k}-r_{k}\right)^{p_{k}+i-j+1} j !} s^{q_{k}-i+j-1} \exp \left(-r_{k} s\right), \\
& E\left[X 1_{\{S>b\}}\right]=\sum_{k=1}^{\infty} \sum_{i=0}^{\beta_{k}-1} C_{k}\left(\begin{array}{c}
\beta_{k}-1 \\
i
\end{array}\right) \frac{(-1)^{i}\left(\alpha_{k}+i\right) ! \Gamma\left(\beta_{k}-i, \delta_{k} b\right)}{\left(\delta_{k}-\gamma_{k}\right)^{\alpha_{k}+i+1} \delta_{k}^{\beta_{k}-i}} \\
& -\sum_{k=1}^{\infty} \sum_{i=0}^{\beta_{k}-1} \sum_{j=0}^{\alpha_{k}+i} C_{k}\left(\begin{array}{c}
\beta_{k}-1 \\
i
\end{array}\right) \frac{(-1)^{i}\left(\alpha_{k}+i\right) ! 2^{\beta_{k}-i} \Gamma\left(\beta_{k}-i+j, \frac{\left(\gamma_{k}+\delta_{k}\right) b}{2}\right)}{\left(\delta_{k}-\gamma_{k}\right)^{\alpha_{k}+i-j+1} j !\left(\gamma_{k}+\delta_{k}\right)^{\beta_{k}-i+j}} \\
& +\sum_{k=1}^{\infty} \sum_{i=0}^{q_{k}-1} \sum_{j=0}^{p_{k}+i} D_{k}\left(\begin{array}{c}
q_{k}-1 \\
i
\end{array}\right) \frac{(-1)^{i}\left(p_{k}+i\right) ! 2^{q_{k}-i} \Gamma\left(q_{k}-i+j, \frac{\left(t_{k}+r_{k}\right) b}{2}\right)}{\left(t_{k}-r_{k}\right)^{p_{k}+i-j+1}\left(t_{k}+r_{k}\right)^{q_{k}-i+j} j !} \\
& -\sum_{k=1}^{\infty} \sum_{i=0}^{q_{k}-1} \sum_{j=0}^{p_{k}+i} D_{k}\left(\begin{array}{c}
q_{k}-1 \\
i
\end{array}\right) \frac{(-1)^{i}\left(p_{k}+i\right) ! \Gamma\left(q_{k}-i+j, r_{k} b\right)}{\left(t_{k}-r_{k}\right)^{p_{k}+i-j+1} r_{k}^{q_{k}-i+j} j !}
\end{aligned}
$$

for $\alpha_{k}>0, \beta_{k}>0, \gamma_{k}>0, \delta_{k}>0, p_{k}>0, q_{k}>0, r_{k}>0, t_{k}>0, x>0$ and $y>0$, provided that $\alpha_{k}, \beta_{k}, p_{k}$ and $q_{k}$ are integers.

Beta Stacey distribution in equation (5.38) of Balakrishnan and Cai (2009):

$$
\begin{aligned}
& f(x, y)=C x^{p-1}(y-x)^{q-1} y^{r} \exp \left(-\alpha y^{\beta}\right) \\
& f_{S}(s)=C 2^{-p} B(p, q) \sum_{k=0}^{\infty} \frac{(-\alpha)^{k}}{k !}{ }_{2} F_{1}\left(-r-\beta k, p ; p+q ; \frac{1}{2}\right) s^{\beta k+p+q+r-1} \\
& F_{S}(s)=C 2^{-p} B(p, q) \sum_{k=0}^{\infty} \frac{(-\alpha)^{k}}{k !(\beta k+p+q+r}{ }_{2} F_{1}\left(-r-\beta k, p ; p+q ; \frac{1}{2}\right) s^{\beta k+p+q+r} \\
& g_{X, S}(s)=C 2^{-p-1} B(p+1, q) \sum_{k=0}^{\infty} \frac{(-\alpha)^{k}}{k !}{ }_{2} F_{1}\left(-r-\beta k, p+1 ; p+q+1 ; \frac{1}{2}\right) s^{\beta k+p+q+r}
\end{aligned}
$$

for $p>0, q>0, r>0, \alpha>0, \beta>0$ and $y>x>0$. 
Mardia (1970)'s bivariate distribution in equation (5.77) of Balakrishnan and Cai (2009):

$$
\begin{aligned}
f(x, y)= & C(\alpha x+1)^{p}(\beta x+1)^{q}(\gamma x+\delta y+1)^{r}, \\
f_{S}(s)= & C \sum_{i=0}^{p} \sum_{j=0}^{q} \sum_{k=0}^{r} \sum_{m=0}^{k}\left(\begin{array}{l}
p \\
i
\end{array}\right)\left(\begin{array}{l}
q \\
j
\end{array}\right)\left(\begin{array}{l}
r \\
k
\end{array}\right)\left(\begin{array}{c}
r \\
m
\end{array}\right) \alpha^{i} \beta^{j} \delta^{m} \gamma^{k-m} \\
& \cdot B(i+k-m+1, j+m+1) s^{i+j+k+1}, \\
F_{S}(s)= & C \sum_{i=0}^{p} \sum_{j=0}^{q} \sum_{k=0}^{r} \sum_{m=0}^{k}\left(\begin{array}{l}
p \\
i
\end{array}\right)\left(\begin{array}{l}
q \\
j
\end{array}\right)\left(\begin{array}{l}
r \\
k
\end{array}\right)\left(\begin{array}{c}
r \\
m
\end{array}\right) \alpha^{i} \beta^{j} \delta^{m} \gamma^{k-m} \\
& \cdot B(i+k-m+1, j+m+1) \frac{s^{i+j+k+2}}{i+j+k+2}, \\
g_{X, S}(s)= & C \sum_{i=0}^{p} \sum_{j=0}^{q} \sum_{k=0}^{r} \sum_{m=0}^{k}\left(\begin{array}{c}
p \\
i
\end{array}\right)\left(\begin{array}{l}
q \\
j
\end{array}\right)\left(\begin{array}{l}
r \\
k
\end{array}\right)\left(\begin{array}{c}
r \\
m
\end{array}\right) \alpha^{i} \beta^{j} \delta^{m} \gamma^{k-m} \\
& \cdot B(i+k-m+2, j+m+1) s^{i+j+k+2}
\end{aligned}
$$

for $\alpha>0, \beta>0, \gamma>0, \delta>0, p>0, q>0, r>0, x>0$ and $y>0$, provided that $p, q$ and $r$ are integers.

Mardia (1970)'s bivariate distribution in equation (5.78) of Balakrishnan and Cai (2009):

$$
\begin{aligned}
& f(x, y)=C x^{p} y^{q}(y-x-1)^{r}, \\
& f_{S}(s)=C 2^{-p-r-1} B(p+1, r+1) s^{q}(s-1)^{p+r+1}{ }_{2} F_{1}\left(-q, p+1 ; p+r+2 ; \frac{s-1}{2 s}\right), \\
& F_{S}(s)=C 2^{-p-r-1} B(p+1, r+1) \sum_{k=0}^{\infty} \frac{(-q)_{k}(p+1)_{k}}{2^{k}(p+r+2)_{k} k !} B_{1-1 / s}(p+r+2+k,-q-p-r-2), \\
& E\left[S 1_{\{S>b\}}\right]=C 2^{-p-r-1} B(p+1, r+1) \sum_{k=0}^{\infty} \frac{(-q)_{k}(p+1)_{k}}{2^{k}(p+r+2)_{k} k !} B_{1 / b}(-p-q-3, p+r+2+k), \\
& g_{X, S}(s)=C 2^{-p-r-1} B(p+2, r+1) s^{q}(s-1)^{p+r+1}{ }_{2} F_{1}\left(-q, p+2 ; p+r+3 ; \frac{s-1}{2 s}\right), \\
& E\left[X 1_{\{S>b\}}\right]=C 2^{-p-r-1} B(p+2, r+1) \sum_{k=0}^{\infty} \frac{(-q)_{k}(p+2)_{k}}{2^{k}(p+r+3)_{k} k !} B_{1 / b}(-p-q-4, p+r+3+k)
\end{aligned}
$$

for $p>0, q>0, r>0, x>0$ and $y>x+1$.

Mardia (1970)'s bivariate gamma distribution in equation (5.81) of Balakrishnan and Cai (2009):

$$
\begin{aligned}
f(x, y)= & C(\alpha x+1)^{p}(\beta x+\gamma y+1)^{q} \exp (-r y), \\
f_{S}(s)= & C \sum_{i=0}^{p} \sum_{j=0}^{q} \sum_{k=0}^{i+j}\left(\begin{array}{c}
p \\
i
\end{array}\right)\left(\begin{array}{c}
q \\
j
\end{array}\right)\left(\begin{array}{c}
i+j \\
k
\end{array}\right) \alpha^{i}(\beta-\gamma)^{j}(-1)^{k} k ! r^{-k-1} s^{i+j-k}(1+\gamma s)^{q-j} \\
& -C \sum_{i=0}^{p} \sum_{j=0}^{q}\left(\begin{array}{c}
p \\
i
\end{array}\right)\left(\begin{array}{c}
q \\
j
\end{array}\right) \alpha^{i}(\beta-\gamma)^{j}(-1)^{i+j}(i+j) ! r^{-i-j-1}(1+\gamma s)^{q-j} \exp (-r s),
\end{aligned}
$$




$$
\begin{aligned}
F_{S}(s)= & C \sum_{i=0}^{p} \sum_{j=0}^{q} \sum_{k=0}^{i+j} \sum_{m=0}^{q-j}\left(\begin{array}{c}
p \\
i
\end{array}\right)\left(\begin{array}{c}
q \\
j
\end{array}\right)\left(\begin{array}{c}
i+j \\
k
\end{array}\right)\left(\begin{array}{c}
q-j \\
m
\end{array}\right) \alpha^{i}(\beta-\gamma)^{j} \gamma^{m}(-1)^{k} k ! r^{-k-1} \frac{s^{i+j-k+m+1}}{i+j-k+m+1} \\
& -C \sum_{i=0}^{p} \sum_{j=0}^{q} \sum_{m=0}^{q-j}\left(\begin{array}{c}
p \\
i
\end{array}\right)\left(\begin{array}{c}
q \\
j
\end{array}\right)\left(\begin{array}{c}
q-j \\
m
\end{array}\right) \alpha^{i}(\beta-\gamma)^{j} \gamma^{m}(-1)^{i+j}(i+j) ! r^{-i-j-m-2} \gamma(m+1, r s), \\
g_{X, S}(s)= & C \sum_{i=0}^{p} \sum_{j=0}^{q} \sum_{k=0}^{i+j}\left(\begin{array}{c}
p \\
i
\end{array}\right)\left(\begin{array}{c}
q \\
j
\end{array}\right)\left(\begin{array}{c}
i+j+1 \\
k
\end{array}\right) \alpha^{i}(\beta-\gamma)^{j}(-1)^{k} k ! r^{-k-1} s^{i+j-k+1}(1+\gamma s)^{q-j} \\
& -C \sum_{i=0}^{p} \sum_{j=0}^{q}\left(\begin{array}{c}
p \\
i
\end{array}\right)\left(\begin{array}{c}
q \\
j
\end{array}\right) \alpha^{i}(\beta-\gamma)^{j}(-1)^{i+j+1}(i+j+1) ! r^{-i-j-2}(1+\gamma s)^{q-j} \exp (-r s)
\end{aligned}
$$

for $\alpha>0, \beta>0, \gamma>0, p>0, q>0, r>0, x>0$ and $y>0$, provided that $p$ and $q$ are integers.

Mardia (1970)'s bivariate distribution in equation (5.79) of Balakrishnan and Cai (2009):

$$
\begin{aligned}
& f(x, y)=C x^{p} y^{q}(x-y-1)^{r}, \\
& f_{S}(s)=C 2^{-q-r-1} B(q+1, r+1) s^{p}(s-1)^{q+r+1}{ }_{2} F_{1}\left(-p, q+1 ; q+r+2 ; \frac{s-1}{2 s}\right), \\
& F_{S}(s)=C 2^{-q-r-1} B(q+1, r+1) \sum_{k=0}^{\infty} \frac{(-p)_{k}(q+1)_{k}}{2^{k}(q+r+2)_{k} k !} B_{1-1 / s}(q+r+2+k,-q-p-r-2), \\
& E\left[S 1_{\{S>b\}}\right]=C 2^{-q-r-1} B(q+1, r+1) \sum_{k=0}^{\infty} \frac{(-p)_{k}(q+1)_{k}}{2^{k}(q+r+2)_{k} k !} B_{1 / b}(-p-q-3, q+r+2+k), \\
& g_{X, S}(s)=C 2^{-q-r-1} B(q+2, r+1) s^{p}(s-1)^{q+r+1}{ }_{2} F_{1}\left(-p, q+2 ; q+r+3 ; \frac{s-1}{2 s}\right), \\
& E\left[X 1_{\{S>b\}}\right]=C 2^{-q-r-1} B(q+2, r+1) \sum_{k=0}^{\infty} \frac{(-p)_{k}(q+2)_{k}}{2^{k}(q+r+3)_{k} k !} B_{1 / b}(-p-q-4, q+r+3+k)
\end{aligned}
$$

for $p>0, q>0, r>0, x>0$ and $x>y+1$.

Bivariate Liouville distribution on page 202 of Balakrishnan and Cai (2009):

$$
\begin{aligned}
& f(x, y)=C x^{\alpha-1} y^{\beta-1} g(x+y), \\
& f_{S}(s)=C B(\alpha, \beta) s^{\alpha+\beta-1} g(s), \\
& F_{S}(s)=C B(\alpha, \beta) \int_{0}^{s} u^{\alpha+\beta-1} g(u) d u, \\
& E\left[S 1_{\{S>b\}}\right]=C B(\alpha, \beta) \int_{b}^{\infty} u^{\alpha+\beta} g(u) d u, \\
& g_{X, S}(s)=C B(\alpha+1, \beta) s^{\alpha+\beta} g(s), \\
& E\left[X 1_{\{S>b\}}\right]=C B(\alpha+1, \beta) \int_{b}^{\infty} u^{\alpha+\beta} g(u) d u
\end{aligned}
$$

for $\alpha>0, \beta>0, x>0$ and $y>0$.

Bivariate Liouville distribution in equation (9.46) of Balakrishnan and Cai (2009):

$$
f(x, y)=C x^{\alpha-1} y^{\beta-1}(x+y)^{-\gamma} g(x+y),
$$




$$
\begin{aligned}
& f_{S}(s)=C B(\alpha, \beta) s^{\alpha+\beta-\gamma-1} g(s), \\
& F_{S}(s)=C B(\alpha, \beta) \int_{0}^{s} u^{\alpha+\beta-\gamma-1} g(u) d u, \\
& E\left[S 1_{\{S>b\}}\right]=C B(\alpha, \beta) \int_{b}^{\infty} u^{\alpha+\beta-\gamma} g(u) d u, \\
& g_{X, S}(s)=C B(\alpha+1, \beta) s^{\alpha+\beta-\gamma} g(s), \\
& E\left[X 1_{\{S>b\}}\right]=C B(\alpha+1, \beta) \int_{b}^{\infty} u^{\alpha+\beta-\gamma} g(u) d u
\end{aligned}
$$

for $\alpha>0, \beta>0, \gamma>0, x>0$ and $y>0$.

Bivariate equilibrium distributions due to Unnikrishnan Nair and Sankaran (2014):

$$
\begin{aligned}
& f(x, y)=\mu^{-1} g(x+y), \\
& f_{S}(s)=\mu^{-1} s g(s), \\
& F_{S}(s)=\mu^{-1} \int_{0}^{s} u g(u) d u, \\
& E\left[S 1_{\{S>b\}}\right]=\mu^{-1} \int_{b}^{\infty} s^{2} g(s) d s, \\
& g_{X, S}(s)=2^{-1} \mu^{-1} s^{2} g(s), \\
& E\left[X 1_{\{S>b\}}\right]=2^{-1} \mu^{-1} \int_{b}^{\infty} s^{2} g(s) d s,
\end{aligned}
$$

where $g(\cdot)$ is the probability density function of a univariate random variable $X$ say and $\mu=E(X)$.

Chacko and Thomas (2007)'s bivariate Pareto distribution:

$$
\begin{aligned}
& f(x, y)=C(x+y)^{-\alpha} \\
& f_{S}(s)=C s^{-\alpha}(s-2 \beta), \\
& F_{S}(s)=\frac{C}{2-\alpha}\left[s^{2-\alpha}-(2 \beta)^{2-\alpha}\right]-\frac{2 \beta C}{1-\alpha}\left[s^{1-\alpha}-(2 \beta)^{1-\alpha}\right], \\
& E\left[S 1_{\{S>b\}}\right]=\frac{C b^{3-\alpha}}{\alpha-3}-\frac{2 \beta C b^{2-\alpha}}{\alpha-2}, \\
& g_{X, S}(s)=2^{-1} C s^{1-\alpha}(s-2 \beta), \\
& E\left[X 1_{\{S>b\}}\right]=\frac{C b^{3-\alpha}}{2(\alpha-3)}-\frac{\beta C b^{2-\alpha}}{\alpha-2}
\end{aligned}
$$

for $\alpha>0, x>\beta>0$ and $y>\beta>0$.

Bivariate Pareto distribution in equation (10.68) of Balakrishnan and Cai (2009):

$$
\begin{aligned}
& f(x, y)=C(a+x+y)^{-\beta} \\
& f_{S}(s)=C s(a+s)^{-\beta} \\
& F_{S}(s)=C\left[\frac{(a+s)^{2-\beta}}{2-\beta}-\frac{a(a+s)^{1-\beta}}{1-\beta}-\frac{a^{2-\beta}}{2-\beta}+\frac{a^{2-\beta}}{1-\beta}\right], \\
& E\left[S 1_{\{S>b\}}\right]=C\left[\frac{(a+b)^{3-\beta}}{\beta-3}+\frac{2 a(a+b)^{2-\beta}}{2-\beta}+\frac{a^{2}(a+b)^{1-\beta}}{\beta-1}\right], \\
& g_{X, S}(s)=2^{-1} C s^{2}(a+s)^{-\beta}, \\
& E\left[X 1_{\{S>b\}}\right]=2^{-1} C\left[\frac{(a+b)^{3-\beta}}{\beta-3}+\frac{2 a(a+b)^{2-\beta}}{2-\beta}+\frac{a^{2}(a+b)^{1-\beta}}{\beta-1}\right]
\end{aligned}
$$


for $a>0, \beta>0, x>0$ and $y>0$.

Bivariate Pareto distribution with equal scale parameters:

$$
\begin{aligned}
& f(x, y)=C x^{\alpha-1} y^{\beta-1}(1+p x+p y)^{-\gamma}, \\
& f_{S}(s)=C B(\alpha, \beta) s^{\alpha+\beta-1}(1+p s)^{-\gamma}, \\
& F_{S}(s)=\frac{C B(\alpha, \beta)}{\alpha+\beta} s^{\alpha+\beta} F_{1}(\gamma, \alpha+\beta ; \alpha+\beta+1 ;-p s), \\
& E\left[S 1_{\{S>b\}}\right]=C B(\alpha, \beta) p^{-\alpha-\beta-1} B_{1 /(1+p b)}(\gamma-\alpha-\beta-1, \alpha+\beta+1), \\
& g_{X, S}(s)=C B(\alpha+1, \beta) s^{\alpha+\beta}(1+p s)^{-\gamma}, \\
& E\left[X 1_{\{S>b\}}\right]=C B(\alpha+1, \beta) p^{-\alpha-\beta-1} B_{1 /(1+p b)}(\gamma-\alpha-\beta-1, \alpha+\beta+1)
\end{aligned}
$$

for $\alpha>0, \beta>0, p>0, \gamma>0, x>0$ and $y>0$.

Bivariate Pareto distribution with unequal scale parameters:

$$
\begin{aligned}
& f(x, y)=C x^{\alpha-1} y^{\beta-1}(1+p x+q y)^{-\gamma}, \\
& f_{S}(s)=C B(\alpha, \beta) s^{\alpha+\beta-1}(1+q s)^{-\gamma}{ }_{2} F_{1}\left(\gamma, \alpha ; \alpha+\beta ; \frac{(q-p) s}{1+q s}\right), \\
& F_{S}(s)=C B(\alpha, \beta) s^{\alpha+\beta} \sum_{k=0}^{\infty} \frac{(\gamma)_{k}(\alpha)_{k}(q-p)^{k} s^{k}}{(\alpha+\beta)_{k} k !(k+\alpha+\beta)} \\
& \quad{ }_{2} F_{1}(k+\gamma, k+\alpha+\beta ; k+\alpha+\beta+1 ;-q s), \\
& E\left[S 1_{\{S>b\}}\right]=C B(\alpha, \beta) q^{-\alpha-\beta-1} \sum_{k=0}^{\infty} \frac{(\gamma)_{k}(\alpha)_{k}(q-p)^{k}}{(\alpha+\beta)_{k} k ! q^{k}} \\
& \quad \cdot B_{1 /(1+q b)}(\gamma-\alpha-\beta-1, k+\alpha+\beta+1), \\
& g_{X, S}(s)=C B(\alpha+1, \beta) s^{\alpha+\beta}(1+q s)^{-\gamma}{ }_{2} F_{1}\left(\gamma, \alpha+1 ; \alpha+\beta+1 ; \frac{(q-p) s}{1+q s}\right), \\
& E\left[X 1_{\{S>b\}}\right]=C B(\alpha+1, \beta) q^{-\alpha-\beta-1} \sum_{k=0}^{\infty} \frac{(\gamma)_{k}(\alpha+1)_{k}(q-p)^{k}}{(\alpha+\beta+1)_{k} k ! q^{k}} \\
& \quad \cdot B_{1 /(1+q b)}(\gamma-\alpha-\beta-1, k+\alpha+\beta+1)
\end{aligned}
$$

for $\alpha>0, \beta>0, \gamma>0, p>0, q>0, x>0$ and $y>0$.

Mixtures of independent Pareto distributions (Lee, 1981):

$$
\begin{aligned}
& f(x, y)=\sum_{k=0}^{\infty} \frac{C_{k} x^{\alpha_{k}-1} y^{\beta_{k}-1}}{\left(1+p_{k} x\right)^{\gamma_{k}}\left(1+q_{k} x\right)^{\delta_{k}}}, \\
& f_{S}(s)=\sum_{k=0}^{\infty} \frac{C_{k} B\left(\alpha_{k}, \beta_{k}\right) s^{\alpha_{k}+\beta_{k}-1}}{\left(1+q_{k} s\right)^{\delta_{k}}} F_{1}\left(\alpha_{k}, \gamma_{k}, \delta_{k}, \alpha_{k}+\beta_{k} ;-p_{k} s, \frac{q_{k} s}{1+q_{k} s}\right) \\
& g_{X, S}(s)=\sum_{k=0}^{\infty} \frac{C_{k} B\left(\alpha_{k}+1, \beta_{k}\right) s^{\alpha_{k}+\beta_{k}}}{\left(1+q_{k} s\right)^{\delta_{k}}} F_{1}\left(\alpha_{k}+1, \gamma_{k}, \delta_{k}, \alpha_{k}+\beta_{k}+1 ;-p_{k} s, \frac{q_{k} s}{1+q_{k} s}\right)
\end{aligned}
$$

for $\alpha_{k}>0, \beta_{k}>0, \gamma_{k}>0, \delta_{k}>0, p_{k}>0, q_{k}>0, x>0$ and $y>0$.

Mixtures of bivariate Pareto distributions with equal scale parameters (Jones, 2002; 
El-Bassiouny and Jones, 2009; Nagar et al., 2009):

$$
\begin{aligned}
& f(x, y)=\sum_{k=0}^{\infty} C_{k} x^{\alpha_{k}-1} y^{\beta_{k}-1}\left(1+p_{k} x+p_{k} y\right)^{-\gamma_{k}}, \\
& f_{S}(s)=\sum_{k=0}^{\infty} C_{k} B\left(\alpha_{k}, \beta_{k}\right) s^{\alpha_{k}+\beta_{k}-1}\left(1+p_{k} s\right)^{-\gamma_{k}}, \\
& F_{S}(s)=\sum_{k=0}^{\infty} \frac{C_{k} B\left(\alpha_{k}, \beta_{k}\right)}{\alpha_{k}+\beta_{k}} s^{\alpha_{k}+\beta_{k}}{ }_{2} F_{1}\left(\gamma_{k}, \alpha_{k}+\beta_{k} ; \alpha_{k}+\beta_{k}+1 ;-p_{k} s\right), \\
& E\left[S 1_{\{S>b\}}\right]=\sum_{k=0}^{\infty} C_{k} B\left(\alpha_{k}, \beta_{k}\right) p^{-\alpha_{k}-\beta_{k}-1} B_{1 /\left(1+p_{k} b\right)}\left(\gamma_{k}-\alpha_{k}-\beta_{k}-1, \alpha_{k}+\beta_{k}+1\right), \\
& g_{X, S}(s)=\sum_{k=0}^{\infty} C_{k} B\left(\alpha_{k}+1, \beta_{k}\right) s^{\alpha_{k}+\beta_{k}}\left(1+p_{k} s\right)^{-\gamma_{k}}, \\
& E\left[X 1_{\{S>b\}}\right]=\sum_{k=0}^{\infty} C_{k} B\left(\alpha_{k}+1, \beta_{k}\right) p^{-\alpha_{k}-\beta_{k}-1} B_{1 /\left(1+p_{k} b\right)}\left(\gamma_{k}-\alpha_{k}-\beta_{k}-1, \alpha_{k}+\beta_{k}+1\right)
\end{aligned}
$$

for $\alpha_{k}>0, \beta_{k}>0, p_{k}>0, \gamma_{k}>0, x>0$ and $y>0$.

Mixtures of bivariate Pareto distributions with unequal scale parameters (Jones, 2002;

El-Bassiouny and Jones, 2009; Nagar et al., 2009):

$$
\begin{aligned}
& f(x, y)= \sum_{k=0}^{\infty} C_{k} x^{\alpha_{k}-1} y^{\beta_{k}-1}\left(1+p_{k} x+q_{k} y\right)^{-\gamma_{k}} \\
& f_{S}(s)= \sum_{k=0}^{\infty} C_{k} B\left(\alpha_{k}, \beta_{k}\right) s^{\alpha_{k}+\beta_{k}-1}\left(1+q_{k} s\right)^{-\gamma_{k}}{ }_{2} F_{1}\left(\gamma_{k}, \alpha_{k} ; \alpha_{k}+\beta_{k} ; \frac{\left(q_{k}-p_{k}\right) s}{1+q_{k} s}\right), \\
& F_{S}(s)= \sum_{k=0}^{\infty} C_{k} B\left(\alpha_{k}, \beta_{k}\right) s^{\alpha_{k}+\beta_{k}} \sum_{i=0}^{\infty} \frac{\left(\gamma_{k}\right)_{i}\left(\alpha_{k}\right)_{i}\left(q_{k}-p_{k}\right)^{i} s^{i}}{\left(\alpha_{k}+\beta_{k}\right)_{i} i !\left(i+\alpha_{k}+\beta_{k}\right)} \\
& \cdot{ }_{2} F_{1}\left(i+\gamma_{k}, i+\alpha_{k}+\beta_{k} ; i+\alpha_{k}+\beta_{k}+1 ;-q_{k} s\right), \\
& E\left[S 1_{\{S>b\}}\right]=\sum_{k=0}^{\infty} C_{k} B\left(\alpha_{k}, \beta_{k}\right) q_{k}^{-\alpha_{k}-\beta_{k}-1} \sum_{i=0}^{\infty} \frac{\left(\gamma_{k}\right)_{i}\left(\alpha_{k}\right)_{i}\left(q_{k}-p_{k}\right)^{i}}{\left(\alpha_{k}+\beta_{k}\right)_{i} i ! q_{k}^{i}} \cdot B_{1 /\left(1+q_{k} b\right)}\left(\gamma_{k}-\alpha_{k}-\beta_{k}-1, i+\alpha_{k}+\beta_{k}+1\right), \\
& g_{X, S}(s)= \sum_{k=0}^{\infty} C_{k} B\left(\alpha_{k}+1, \beta_{k}\right) s^{\alpha_{k}+\beta_{k}}\left(1+q_{k} s\right)^{-\gamma_{k}}{ }_{2} F_{1}\left(\gamma_{k}, \alpha_{k}+1 ; \alpha_{k}+\beta_{k}+1 ; \frac{\left(q_{k}-p_{k}\right) s}{1+q_{k} s}\right), \\
& E\left[X 1_{\{S>b\}}\right]=\sum_{k=0}^{\infty} C_{k} B\left(\alpha_{k}+1, \beta_{k}\right) q_{k}^{-\alpha_{k}-\beta_{k}-1} \sum_{i=0}^{\infty} \frac{\left(\gamma_{k}\right)_{i}\left(\alpha_{k}+1\right)_{i}\left(q_{k}-p_{k}\right)^{i}}{\left(\alpha_{k}+\beta_{k}+1\right)_{i} i ! q_{k}^{i}} \\
& \quad \cdot B_{1 /\left(1+q_{k} b\right)}\left(\gamma_{k}-\alpha_{k}-\beta_{k}-1, i+\alpha_{k}+\beta_{k}+1\right)
\end{aligned}
$$

for $\alpha_{k}>0, \beta_{k}>0, \gamma_{k}>0, p_{k}>0, q_{k}>0, x>0$ and $y>0$.

\section{Generalized bivariate Pareto distribution:}

$$
f(x, y)=\frac{C x^{\alpha-1} y^{\beta-1}}{(1+p x+q y+r x y)^{\delta}}
$$




$$
\begin{aligned}
& f_{S}(s)=C B(\alpha, \beta)(-r)^{-\delta}(u v)^{-\delta} s^{\alpha+\beta-1} F_{1}\left(\alpha, \delta, \delta, \alpha+\beta ; \frac{s}{u}, \frac{s}{v}\right) \\
& g_{X, S}(s)=C B(\alpha+1, \beta)(-r)^{-\delta}(u v)^{-\delta} s^{\alpha+\beta} F_{1}\left(\alpha+1, \delta, \delta, \alpha+\beta+1 ; \frac{s}{u}, \frac{s}{v}\right)
\end{aligned}
$$

for $\alpha>0, \beta>0, \gamma>0, \delta>0, p>0, q>0, r>0, x>0$ and $y>0$, where $u=\frac{p-q+r s}{2 r}+$ $\sqrt{\frac{1+q s}{r}+\frac{(p-q+r s)^{2}}{4 r^{2}}}$ and $v=\frac{p-q+r s}{2 r}-\sqrt{\frac{1+q s}{r}+\frac{(p-q+r s)^{2}}{4 r^{2}}}$.

\section{Lee and Cha (2014)'s bivariate distribution:}

$$
\begin{aligned}
f(x, y)= & \left\{\begin{array}{cl}
C(x+\alpha)^{\gamma}(y+\beta)^{\delta}, & \text { if } x<y, \\
D(x+t)^{v}(y+u)^{w}, & \text { if } x>y,
\end{array}\right. \\
f_{S}(s)= & 3^{-1} C \alpha^{\gamma} s^{-1}(s+\beta)^{\delta} F_{1}\left(1,-\gamma,-\delta, 2 ;-\frac{s}{2 \alpha}, \frac{s}{2(\alpha+\beta)}\right) \\
& +3^{-1} D s^{-1}\left(\frac{s}{2}+t\right)^{v}\left(\frac{s}{2}+u\right)^{w} F_{1}\left(1,-v,-w, 2 ;-\frac{s}{s+2 t}, \frac{s}{s+2 u}\right), \\
g_{X, S}(s)= & 3^{-1} C \alpha^{1+\gamma} s^{-1}(s+\beta)^{\delta} F_{1}\left(1,-1-\gamma,-\delta, 2 ;-\frac{s}{2 \alpha}, \frac{s}{2(\alpha+\beta)}\right) \\
& -3^{-1} C \alpha^{1+\gamma} s^{-1}(s+\beta)^{\delta} F_{1}\left(1,-\gamma,-\delta, 2 ;-\frac{s}{2 \alpha}, \frac{s}{2(\alpha+\beta)}\right) \\
& +3^{-1} D s^{-1}\left(\frac{s}{2}+t\right)^{1+v}\left(\frac{s}{2}+u\right)^{w} F_{1}\left(1,-1-v,-w, 2 ;-\frac{s}{s+2 t}, \frac{s}{s+2 u}\right) \\
& -3^{-1} D t s^{-1}\left(\frac{s}{2}+t\right)^{v}\left(\frac{s}{2}+u\right)^{w} F_{1}\left(1,-v,-w, 2 ;-\frac{s}{s+2 t}, \frac{s}{s+2 u}\right)
\end{aligned}
$$

for $\alpha>0, \beta>0, t>0, u>0, \delta<0, v<0, x>0$ and $y>0$.

\section{Truncated bivariate normal distribution:}

$$
\begin{aligned}
f(x, y)= & C \exp \left(-\alpha x^{2}-\beta y^{2}-\gamma x-\delta y-\theta x y\right) \\
f_{S}(s)= & C \sqrt{\pi} p^{-1 / 2} \exp \left(-\beta s^{2}-\delta s+\frac{q^{2}}{4 p}\right)\left[\Phi\left(\sqrt{2 p} s-(2 p)^{-1 / 2} q\right)-\Phi\left(-(2 p)^{-1 / 2} q\right)\right], \\
g_{X, S}(s)= & 2^{-1} C \sqrt{\pi} p^{-3 / 2} q \exp \left(-\beta s^{2}-\delta s+\frac{q^{2}}{4 p}\right)\left[\Phi\left(\sqrt{2 p} s-(2 p)^{-1 / 2} q\right)-\Phi\left(-(2 p)^{-1 / 2} q\right)\right] \\
& -2^{-1} C p^{-1} \exp \left(-\beta s^{2}-\delta s+\frac{q^{2}}{4 p}\right)\left[\exp \left(-p s^{2}+q s\right)-1\right]
\end{aligned}
$$

for $\alpha>0, \beta>0,-\infty<\gamma<\infty,-\infty<\delta<\infty,-\infty<\theta<\infty, x>0$ and $y>0$, where $p=\alpha+\beta-\theta$ and $q=2 \beta s-\gamma+\delta-\theta s$.

\section{References}

[1] Arnold, B. C. and Strauss, D. (1991). Bivariate distributions with conditionals in prescribed exponential families. Journal of the Royal Statistical Society, B, 53, 365-375.

[2] Balakrishnan, N. and Lai, C. D. (2009). Continuous Bivariate Distributions, second edition. Springer Verlag, New York. 
[3] Barges, M., Cossette, H. and Marceau, E. (2009). TVaR-based capital allocation with copulas. Insurance: Mathematics and Economics, 45, 348-361.

[4] Becker, P. J. and Roux, J. J. J. (1981). A bivariate extension of the gamma distribution. South African Statistical Journal, 15, 1-12.

[5] Block, H. W. and Basu, A. P. (1976). A continuous bivariate exponential distribution. Journal of the American Statistical Association, 64, 1031-1037.

[6] Chacko, M. and Thomas, P. Y. (2007). Estimation of a parameter of bivariate Pareto distribution by ranked set sampling. Journal of Applied Statistics, 34, 703-714.

[7] Cheriyan, K. C. (1941). A bivariate correlated gamma-type distribution function. Journal of the Indian Mathematical Society, 5, 133-144.

[8] Chiragiev, A. and Landsman, Z. (2007). Multivariate Pareto portfolios: TCE based capital allocation and divided differences. Scandinavian Actuarial Journal, 261-280.

[9] Cossette, H., Cote, M. -P., Marceau, E. and Moutanabbir, K. (2013). Risk measures and capital allocation using the Farlie-Gumbel-Morgenstern copula. Insurance: Mathematics and Economics, 52, 560-572.

[10] Cossette, H., Mailhot, M. and Marceau, E. (2012). TVaR-based capital allocation for multivariate compound distributions. Insurance: Mathematics and Economics, 50, 247-256.

[11] Cossette, H., Marceau, E. and Perreault, S. (2015). On two families of bivariate distributions with exponential marginals: Aggregation and capital allocation. Insurance: Mathematics and Economics, 64, 214-224.

[12] Crovelli, R. A. (1973). A bivariate precipitation model. Journal of the American Meteorological Society, 1, 130-134.

[13] Dhaene, J., Henrard, L., Landsman, Z., Vandendorpe, A. and Vanduffel, S. (2008). Some results on the CTE-based capital allocation rule. Insurance: Mathematics and Economics, $42,855-863$.

[14] Dussauchoy, A. and Berland, R. (1975). A multivariate gamma-type distribution whose marginal laws are gamma, and which has a property similar to a characteristic property of the normal case. In: A Modern Course on Distributions in Scientific Work, volume I: Models and Structures, editors G. P. Patil, S. Kotz and J. K. Ord, pp. 319-328, Reidel, Dordrecht.

[15] El-Bassiouny, A. H. and Jones, M. C. (2009). A bivariate $F$ distribution with marginals on arbitrary numerator and denominator degrees of freedom, and related bivariate beta and $t$ distributions. Statistical Methods and Applications, 18, 465-481.

[16] Furman, E. and Landsman, Z. (2005). Risk capital decomposition for a multivariate dependent gamma portfolio. Insurance: Mathematics and Economics, 37, 635-649.

[17] Furman, E. and Landsman, Z. (2008). Economic capital allocations for non-negative portfolios of dependent risks. ASTIN Bulletin, 38, 601-619.

[18] Jones, M. C. (2002). A dependent bivariate $t$ distribution with marginals on different degrees of freedom. Statistics and Probability Letters, 56, 163-170. 
[19] Landsman, Z. M. and Valdez, E. A. (2003). Tail conditional expectations for elliptical distributions. North American Actuarial Journal, 7, 55-71.

[20] Lawrance, A. J. and Lewis, P. A. W. (1980). The exponential autoregressive-moving average $\operatorname{EARMA}(p, q)$ process. Journal of the Royal Statistical Society, B, 42, 150-161.

[21] Lee, H. and Cha, J. H. (2014). On construction of general classes of bivariate distributions. Journal of Multivariate Analysis, 127, 151-159.

[22] Lee, P. A. (1981). The correlated bivariate inverted beta distribution. Biometrical Journal, 23, 693-703.

[23] Mardia, K. V. (1970). Families of Bivariate Distributions. Griffin, London.

[24] Mirhosseini, S. M., Amini, M., Kundu, D. and Dolati, A. (2015). On a new absolutely continuous bivariate generalized exponential distribution. Statistical Methods and Applications, $24,61-83$.

[25] Mohsin, M., Gebhardt, A., Pilz, J. and Spock, G. (2013). A new bivariate gamma distribution generated from functional scale parameter with application to drought data. Stochastic Environmental Research and Risk Assessment, 27, 1039-1054.

[26] Nadarajah, S. and Gupta, A. K. (2006). Some bivariate gamma distributions. Applied Mathematics Letters, 19, 767-774.

[27] Nagar, D. K., Bran-Cardona, P. A. and Gupta, A. K. (2009). Multivariate generalization of the hypergeometric function type I distribution. Acta Applicande Mathematicae, 105, 111122 .

[28] Nagar, D. K. and Sepulveda-Murillo, F. H. (2011). Properties of the bivariate confluent hypergeometric function kind 1 distribution. Revista de la Union Matematica Argentina, 52, $11-21$.

[29] Panjer, H. H. (2002). Measurement of risk, solvency requirements and allocation of capital within financial conglomerates. Research Report, Institute of Insurance and Pension Research, University of Waterloo.

[30] Tasche, D. (1999). Risk contributions and performance measurement. Working Paper, Technische Universitatt Mnchen.

[31] Unnikrishnan Nair, N. and Sankaran, P. G. (2014). Modelling lifetimes with bivariate Schurconstant equilibrium distributions from renewal theory. Metron, 72, 331-349. 